\title{
Some Comments on the Inverse Problem of Pure Point Diffraction
}

\author{
Venta Terauds and Michael Baake
}

\begin{abstract}
In a recent paper [11], Lenz and Moody presented a method for constructing families of real solutions to the inverse problem for a given pure point diffraction measure. Applying their technique and discussing some possible extensions, we present, in a non-technical manner, some examples of homometric structures.
\end{abstract}

\section{Introduction}

Kinematic diffraction is concerned with the Fourier transform $\widehat{\gamma}$ of the autocorrelation $\gamma$ of a given structure, the latter described by a measure $\omega$, which is usually assumed to be translation bounded; see [2] for a recent summary of the state of affairs. Of particular relevance (for crystals and quasicrystals, say) are systems with pure point (or pure Bragg) diffraction, i.e., those where $\widehat{\gamma}$ is a pure point measure.

In considering the inverse problem, namely the problem of determining which structure or structures could have produced a given diffraction, one is naturally led to the concept of homometry, where structures that give rise to the same diffraction measure are said to be homometric. Via the autocorrelation measure, homometry is well-defined for certain classes of measures, and, accordingly, also for objects such as point sets and tilings (an overview of these concepts is given in [3, Ch. 9.6]). Various methods have been used (see [1, 3, 7, 8] and references therein) to construct different objects (mainly Dirac combs) that are homometric. However, a way of finding all objects with a given diffraction, which we shall refer to as the diffraction solution class of a given diffraction measure, has long remained elusive.

In [11], Lenz and Moody present a method for abstractly parametrising the real solution class of a given pure point diffraction measure. Their approach is based on the Halmos-von Neumann theorem in conjunction with concepts from the theory of

Fakultät für Mathematik, Universität Bielefeld, Postfach 100131, 33501 Bielefeld, Germany

. Venta Terauds: terauds@math.uni-bielefeld.de

Michael Baake: mbaake@math.uni-bielefeld.de 
(stochastic) point processes. The objects constructed via their method are in many cases measures, but as we shall see, even for a very simple periodic diffraction, one may construct objects with that diffraction that generically fail to be measures. Thus many open questions remain, in particular whether (or in what sense) non-measure solutions have a reasonable physical interpretation.

The mathematical formalism behind the construction method in [11] is based on a theory of (generalised) point processes and is quite formidable. In essence, it justifies the use of the lower path of the Wiener diagram

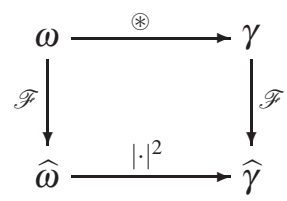

in the reverse direction, where $\mathscr{F}$ denotes Fourier transform and $\circledast$ the volumeaveraged (or Eberlein) convolution (so that $\gamma=\omega \circledast \widetilde{\omega}$; compare [10, 2]). In particular, for a diffraction measure of the form $\widehat{\gamma}=\sum_{k \in L}|A(k)|^{2} \delta_{k}$, with $L$ a countable set, one may (formally, but consistently) invert the left as well as the bottom arrow of the diagram (the latter interpreted as $|\cdot|^{2}$ being applied to the coefficients or 'intensities' $I(k)=|A(k)|^{2}$ individually) to construct a measure (or at least a tempered distribution) $\omega$ with diffraction $\widehat{\gamma}$.

It is well known that for certain measures (for example the Dirac comb of a lattice or, more generally, of a crystallographic structure [9, 10, 6]), one may proceed via the lower route in the forward, and thus also the reverse, direction in this way. However, the results of Lenz and Moody apply in a much more general situation.

The purpose of this brief contribution is to present, without the point process formalism of [11], some examples of object classes that display the same diffraction. Unless stated otherwise, all measures presented below are measures on the real line $\mathbb{R}$, which means that we illustrate everything with one-dimensional examples.

\section{A simple diffraction measure with simple origins}

A diffraction measure, that is, a measure that represents the diffraction of some (physical) structure, must be real, positive and inversion symmetric. By pure point, we mean that the measure can be written as $\widehat{\gamma}=\sum_{k \in L} I(k) \delta_{k}$, with a countable set $L$ (which may be finite) and locally summable intensities $I(k)>0$. Let us begin then, with the 'simplest possible' pure point diffraction measure.

Example 1. Let $\widehat{\gamma}=\delta_{0}$. Proceeding backwards through the Wiener diagram via the 'bottom' route, we gain $\widehat{\omega}=A(0) \delta_{0}$, where, applying the method of [11], we must have $A(0)=1$. Since $\widehat{\lambda}=\delta_{0}$ and $\widehat{\delta_{0}}=\lambda$, where $\lambda$ is Lebesgue measure, we have $\omega=\lambda$ and the real diffraction solution class of $\delta_{0}$ is $\{\lambda,-\lambda\}$ (as it is clear that $-\omega$ is homometric to $\omega$ ). Of course, observing that $\lambda \circledast \widetilde{\lambda}=\lambda$ gives us the same thing via the top route in the Wiener diagram. 
It is not hard to deduce a bit more here: For $u \in \mathbb{S}^{1}$, the unit circle, we have $u \lambda \circledast \widetilde{u \lambda}=u \bar{u} \lambda=\lambda$, and so, via the top route, we see that $\left\{u \lambda \mid u \in \mathbb{S}^{1}\right\}$ is contained in the (complex) diffraction solution class of $\delta_{0}$. Are there further measures with diffraction $\delta_{0}$ ? Well, any measure of the form $\omega+\mu$, with $\mu$ a finite measure on $\mathbb{R}$, has the same diffraction as $\omega$, as adding a finite measure to $\omega$ does not change the autocorrelation. This is well-known [9, 3] and a 'trivial' degree of freedom; in the framework of [11], the point process for $\omega+\mu$ is the same as that for $\omega$.

In this example, we have a good idea of what the complex diffraction solution class is, and are certain that the real diffraction solution class contains only measures. In the next section, we shall see that the real diffraction solution class of a nice, periodic measure like $\delta_{\mathbb{Z}}$ contains both measures and non-measures. In fact, as will be shown in [12], the only pure point diffraction measures whose real solution class consists solely of measures are those supported on a finite set of points.

\section{A lattice diffraction measure with all kinds of origins}

Let us now consider measures with diffraction $\widehat{\gamma}=\delta_{\mathbb{Z}}=\sum_{k \in \mathbb{Z}} \delta_{k}$. According to the Wiener diagram, objects $\omega$ with the diffraction $\delta_{\mathbb{Z}}$ must have the (possibly formal) Fourier transform $\widehat{\omega}=\sum_{k \in \mathbb{Z}} A(k) \delta_{k}$, with $|A(k)|=1$ for all $k$. In the setting of [11], we have the further conditions that $A(0)=1$ and $A(-k)=\overline{A(k)}$ for all $k$, and one constructs different objects with diffraction $\delta_{\mathbb{Z}}$ simply by choosing different sets of compliant coefficients $\{A(k) \mid k \in \mathbb{Z}\}$. An interpretation of $\omega$ and $\widehat{\omega}$ might need the theory of tempered distributions and their relations with measures.

Example 2. Choosing $A(k)=1$ for all $k$, one gains $\omega=\delta_{\mathbb{Z}}$, as follows from the Poisson summation formula $\widehat{\delta_{\mathbb{Z}}}=\delta_{\mathbb{Z}}$; compare [5, 3].

Example 3. By splitting the set $\mathbb{Z}$ into subsets $n \mathbb{Z}, n \mathbb{Z}+1, \ldots, n \mathbb{Z}+(n-1)$, and choosing coefficients appropriately, one may construct an $n$-periodic measure with diffraction $\delta_{\mathbb{Z}}$. For example, to construct a 4-periodic measure, let

$$
A(k)= \begin{cases}1, & k \in 4 \mathbb{Z}, \\ \alpha, & k \in 4 \mathbb{Z}+1, \\ e, & k \in 4 \mathbb{Z}+2, \\ \bar{\alpha}, & k \in 4 \mathbb{Z}+3,\end{cases}
$$

where $e= \pm 1$ and $\alpha \in \mathbb{S}^{1}$. Note that this exhausts all possibilities for compliant coefficients for this four-way splitting. We have

$$
\widehat{\omega_{\alpha}^{ \pm}}=\left(\delta_{0}+\alpha \delta_{1}+\bar{\alpha} \delta_{-1} \pm \delta_{2}\right) * \delta_{4 \mathbb{Z}} .
$$

Then, writing $\alpha=e^{2 \pi i t_{\alpha}}$ and defining $\chi_{s}(t):=e^{2 \pi \text { ist }}$ for $t, s \in \mathbb{R}$, we obtain 


$$
\begin{aligned}
\omega_{\alpha}^{ \pm} & =\frac{1}{4}\left(\chi_{0}+\alpha \chi_{1}+\bar{\alpha} \chi_{-1} \pm \chi_{2}\right) \delta_{\frac{1}{4} \mathbb{Z}} \\
& =\frac{1}{4} \sum_{k \in \mathbb{Z}}\left(1+2 \cos \left(2 \pi\left(t_{\alpha}+\frac{k}{4}\right)\right) \pm \cos (\pi k)\right) \delta_{\frac{k}{4}},
\end{aligned}
$$

where we have used the the Poisson summation formula and the identity $\widehat{\mu * v}=\widehat{\mu} \widehat{v}$. That is, we have a measure of the form $\omega_{\alpha}^{ \pm}=\left(a \delta_{0}+b \delta_{\frac{1}{4}}+c \delta_{\frac{1}{2}}+d \delta_{\frac{3}{4}}\right) * \delta_{\mathbb{Z}}$. For example, choosing $\alpha \in\{0, i, 1,-i\}$, we get $\omega_{\alpha}^{ \pm}$according to the following table.

\begin{tabular}{|c|l|l|}
\hline$\omega_{\alpha}^{ \pm}$ & $e=1$ & $e=-1$ \\
\hline$t_{\alpha}=0$ & $\delta_{\mathbb{Z}}$ & $\frac{1}{2}\left(\delta_{0}+\delta_{\frac{1}{4}}-\delta_{\frac{1}{2}}+\delta_{\frac{3}{4}}\right) * \delta_{\mathbb{Z}}$ \\
\hline$t_{\alpha}=\frac{1}{4}$ & $\frac{1}{2}\left(\delta_{0}-\delta_{\frac{1}{4}}+\delta_{\frac{1}{2}}+\delta_{\frac{3}{4}}\right) * \delta_{\mathbb{Z}}$ & $\delta_{\frac{3}{4}} * \delta_{\mathbb{Z}}$ \\
\hline$t_{\alpha}=\frac{1}{2}$ & $\delta_{\frac{1}{2}} * \delta_{\mathbb{Z}}$ & $\frac{1}{2}\left(-\delta_{0}+\delta_{\frac{1}{4}}+\delta_{\frac{1}{2}}+\delta_{\frac{3}{4}}\right) * \delta_{\mathbb{Z}}$ \\
\hline$t_{\alpha}=\frac{3}{4}$ & $\frac{1}{2}\left(\delta_{0}+\delta_{\frac{1}{4}}+\delta_{\frac{1}{2}}-\delta_{\frac{3}{4}}\right) * \delta_{\mathbb{Z}}$ & $\delta_{\frac{1}{4}} * \delta_{\mathbb{Z}}$ \\
\hline
\end{tabular}

It is easy to verify (along the top route of the Wiener diagram) that these measures do indeed all have autocorrelation $\gamma=\delta_{\mathbb{Z}}$ and thus diffraction $\widehat{\gamma}=\delta_{\mathbb{Z}}$. All elements constructed are pure point measures; compare [8] for similar examples.

Example 4. We now construct a measure that is not itself pure point, but has diffraction $\delta_{\mathbb{Z}}$. Take $A(0)=1$ and $A(k)=-1$ for all $k \in \mathbb{Z} \backslash\{0\}$. Then we have

$$
\widehat{\omega}=\delta_{0}-\sum_{k \in \mathbb{Z} \backslash\{0\}} \delta_{k}=2 \delta_{0}-\delta_{\mathbb{Z}}
$$

and see that $\omega=2 \lambda-\delta_{\mathbb{Z}}$ is a member of the (real) diffraction solution class of $\delta_{\mathbb{Z}}$.

Recalling that $\lambda \circledast \delta_{\mathbb{Z}}=\lambda$, one can easily verify the above example and construct similar ones via a little trial and error and the top route of the Wiener diagram. For example, via a quick calculation with the Eberlein convolution, one can see that the diffraction of $\delta_{\mathbb{Z}}-(1+\mathrm{i}) \lambda$ is also $\delta_{\mathbb{Z}}$, while $\delta_{\mathbb{Z}}-\lambda$ has diffraction $\delta_{\mathbb{Z} \backslash\{0\}}$. Such a trial and error method, however, would probably not lead to the following more elaborate system.

Example 5. To construct something a little different, we use an aperiodic set suggested by the period doubling sequence, which is limit-periodic; compare [3, 6]. From the set

$$
\Lambda=\bigcup_{n \geq 0}\left(2.4^{n} \mathbb{Z}+\left(4^{n}-1\right)\right)
$$

of left endpoints of ' $a$ 's in this sequence [4], we form the symmetric set

$$
\Delta:=\Lambda \cup(-\Lambda)=2 \mathbb{Z} \cup \bigcup_{n \geq 1}^{\dot{s}} \Delta_{n}
$$

where $\Delta_{n}:=\left(2.4^{n} \mathbb{Z}+\left(4^{n}-1\right)\right) \cup\left(2.4^{n} \mathbb{Z}+\left(1-4^{n}\right)\right)$ for $n \geq 1$ and $\dot{\cup}$ denotes the disjoint union of sets. Then, we define $A(k)=1$ for $k \in \Delta$ and $A(k)=-1$ for $k \notin \Delta$. 
Due to the symmetry of $\Delta$, these coefficients satisfy the conjugacy condition given above. We get

$$
\widehat{\omega}=\sum_{k \in \Delta} \delta_{k}-\sum_{k \in \mathbb{Z} \backslash \Delta} \delta_{k}=2 \delta_{\Delta}-\delta_{\mathbb{Z}}
$$

and thus $\omega=2 \widehat{\delta_{\Delta}}-\delta_{\mathbb{Z}}$. Now, we have

$$
\delta_{L}=\delta_{2 \mathbb{Z}}+\sum_{n \geq 1} \delta_{\Delta_{n}}=\delta_{2 \mathbb{Z}}+\sum_{n \geq 1} \delta_{2 \cdot 4^{n} \mathbb{Z}} *\left(\delta_{4^{n}-1}+\delta_{1-4^{n}}\right)
$$

and hence may use the Poisson summation formula to derive the formal expression

$$
\begin{aligned}
\widehat{\delta_{\Delta}} & =\frac{1}{2} \delta_{\frac{\mathbb{Z}}{2}}+\sum_{n \geq 1} \frac{\bar{\chi}_{4^{n}-1}+\bar{\chi}_{1-4^{n}}}{2.4^{n}} \delta_{\frac{\mathbb{Z}}{2 \cdot 4^{n}}} \\
& =\frac{1}{2} \delta_{\frac{\mathbb{Z}}{2}}+\sum_{n \geq 1} \frac{\cos \left(2 \pi\left(4^{n}-1\right) k\right)}{4^{n}} \delta_{\frac{\mathbb{Z}}{2 \cdot 4^{n}}} .
\end{aligned}
$$

As $\delta_{\Delta}$ is a translation bounded measure, $\widehat{\delta_{\Delta}}$ is a tempered distribution. It is not, however, a measure, as it is easy to find compact sets $K \subseteq \mathbb{R}$ (for example, take $\left.K=\left[0, \frac{1}{4}\right]\right)$ such that $\left|\widehat{\delta}_{\Delta}\right|(K)$ is infinite. So, we have a tempered distribution, $\omega$, that is not a measure, but nevertheless has diffraction $\delta_{\mathbb{Z}}$.

Of course, one can construct many such 'non-measures' (in [12], via a theorem of Cordoba [5], it is shown that the homometry class of $\delta_{\mathbb{Z}}$ contains uncountably many such objects), but this does not shed much light on the physical relevance of such constructions. A little more insight may be gained by noting that (in this case at least) our constructed distribution is the limit (in the weak- $*$ topology on the space $\mathscr{S}^{\prime}(\mathbb{R})$ of tempered distributions) of a sequence of measures over $\mathbb{R}$.

For $\varepsilon>0$, define

$$
\rho_{\varepsilon}:=\frac{1}{2} \delta_{\frac{\mathbb{Z}}{2}}+\sum_{n \geq 1} \frac{\cos \left(2 \pi\left(4^{n}-1\right) k\right)}{(4+\varepsilon)^{n}} \delta_{\frac{\mathbb{Z}}{2 \cdot 4^{n}}} .
$$

A short calculation reveals that, for $\varepsilon>0,\left|\rho_{\varepsilon}\right|(K)$ is finite for all compact sets $K \subseteq \mathbb{R}$, so that $\rho_{\varepsilon}$ is indeed a measure (it is even translation-bounded). Thus $\omega_{\varepsilon}:=$ $2 \rho_{\varepsilon}-\delta_{\mathbb{Z}}$ is also a measure. Moreover, for all Schwartz functions $g \in \mathscr{S}(\mathbb{R})$, one has $\omega_{\varepsilon}(g) \rightarrow \omega(g)$ as $\varepsilon \rightarrow 0^{+}$. This is a standard approach in Fourier analysis to enforce convergence of the series, which is sometimes referred to as 'regularisation' in physics. Such objects can still be given a reasonable physical meaning.

\section{Further remarks}

The method of [11] may only be applied to diffraction measures $\widehat{\gamma}$ that are 'backward transformable', meaning that the (inverse) Fourier transform, $\gamma$ (that is, the 
autocorrelation), is again a measure. Further contemplation of the previous example, however, makes this condition seem a little too restrictive. Using the scheme, we constructed the object $\omega=2 \widehat{\delta_{\Delta}}-\delta_{\mathbb{Z}}$, which is not a measure, but is the weak-* limit (in $\mathscr{S}^{\prime}(\mathbb{R})$ ) of measures. The object $\widehat{\delta_{\Delta}}$ is also a non-measure weak-* limit of measures, but does not have the good fortune, as $\omega$ does, to have a measure-valued autocorrelation, so it is not covered by the scheme.

Presuming that one may extend the method of [11] to admit this case (or, in other words, that one may proceed via the lower path in the Wiener diagram to calculate the diffraction), one has $\omega=\widehat{\delta_{\Delta}}$, with $\widehat{\omega}=\delta_{\Delta}$, and thus diffraction $\widehat{\gamma}=\delta_{\Delta}$. The measure $\delta_{\Delta}$ is positive, inversion symmetric and translation bounded, so has almost all of the properties that one expects from a diffraction measure.

The natural next step is to understand the classes of objects for which one may define an autocorrelation (and thus a diffraction). The framework of [11] is applicable in the more general setting of a locally compact Abelian group. However, if one considers only objects in $\mathbb{R}^{d}$, these initial examples suggest that consideration of tempered distributions that are the weak-* limits of measures may be a good place to begin.

Acknowledgements This work was supported by the German Research Council (DFG), via the CRC 701, and by the $\mathrm{RCM}^{2}$, at the University of Bielefeld.

\section{References}

1. M. Baake and U. Grimm, Homometric model sets and window covariograms, Z. Krist. 222 (2007), 54-58.

2. M. Baake and U. Grimm, Mathematical diffraction of aperiodic structures, Chem. Soc. Rev. 41 (2012), 6821-6843.

3. M. Baake and U. Grimm, Theory of Aperiodic Order: A Mathematical Invitation, Cambridge University Press, Cambridge, in preparation.

4. M. Baake and R.V. Moody, Weighted Dirac combs with pure point diffraction, J. Reine Angew. Math. (Crelle) 573 (2004), 61-94.

5. A. Córdoba, Dirac combs, Lett. Math. Phys. 17 (1989), 191-196.

6. F. Gähler and R. Klitzing, The diffraction pattern of self-similar tilings, in The Mathematics of Long-Range Aperiodic Order, ed. R.V. Moody, NATO ASI Series C489, Kluwer, Dordrecht, 1995, pp. 141-174.

7. U. Grimm and M. Baake, Homometric point sets and inverse problems, Z. Krist. 223 (2008), 777-781.

8. F.A. Grünbaum and C.C. Moore, The use of higher-order invariants in the determination of generalized Patterson cyclotomic sets, Acta Cryst. A 51 (1995), 310-323.

9. A. Hof, On diffraction by aperiodic structures, Commun. Math. Phys. 169 (1995), 25-43.

10. A. Hof, Diffraction by aperiodic structures, in The Mathematics of Long-Range Aperiodic Order, ed. R.V. Moody, NATO ASI Series C489, Kluwer, Dordrecht, 1995, pp. 239-268.

11. D. Lenz and R.V. Moody, Stationary processes with pure point diffraction, preprint arXiv: 1111.3617

12. V. Terauds, The inverse problem of pure point diffraction - examples and open questions, in preparation. 